\title{
Statistical multi-path exposure method for assessing the whole-body SAR in a heterogeneous human body model in a realistic environment
}

\author{
Günter Vermeeren, Wout Joseph, Luc Martens*
}

April 2013

\begin{abstract}
Assessing the whole-body absorption in a human in a realistic environment requires a statistical approach covering all possible exposure situations. This paper describes the development of a statistical multi-path exposure method for heterogeneous realistic human body models. The method is applied for the 6-year-old virtual family boy exposed to the GSM downlink at $950 \mathrm{MHz}$. It is shown that the whole-body SAR does not differ significantly over the different environments at an operating frequency of $950 \mathrm{MHz}$. Furthermore, the whole-body SAR in the virtual family boy exceeds the worst-case single incident plane-wave exposure and the ICNIRP basic restrictions for $3.6 \%$ and $0.3 \%$ of the exposure samples, respectively, for an incident power density equal to the ICNIRP reference level at $950 \mathrm{MHz}$. The homogeneous spheroid with the dielectric properties of head suggested by IEC underestimates the absorption compared to realistic human body models. Moreover, the variation of the whole-body SAR for realistic human body models is larger than for homogeneous spheroid models. This is mainly due to the heterogeneity of the tissues and the irregular shape of the realistic human body model in contrary to the homogeneous spheroid human body models.
\end{abstract}

\section{Introduction}

The advent of broadcasting and wireless communication systems increased enormously the exposure of people to radio-frequency ( $\mathrm{RF}$ ) electromagnetic fields (EMF). The international committee on nonionizing radiation protection (ICNIRP) and the institute of electric and electronic engineers (IEEE) issued guidelines [ICNIRP, 1998; IEEE SCC39, 2005 ] to protect people against adverse health effects of EMF exposure. The basic quantities for the limits of RF EMF defined in these guidelines are the whole-body and localized absorption of electromagnetic energy in the human body in terms of Specific Absorption Rate (SAR). Unfortunately, it is not possible to measure the absorption in a living

${ }^{*}$ Department of Information Technology, Ghent University/Interdisciplinary Institute for Broadband Technology (IBBT), Ghent, Belgium person. In contrary to absorption, the incident field levels are easy to measure. Therefore, reference levels (RL) for the incident fields are derived from the basic restrictions (BR) based on measurements and numerical computations using a spheroid homogeneous phantom exposed to a horizontally incident plane wave [ICNIRP 1998].

The assessment of the absorption in a real environment based on the measured field levels is a very difficult, complex, and time-consuming task as a huge amount of exposure situations need to be computed. On the one hand, the RF absorption in a human body depends on the external and internal morphology [Conil et al., 2008; Dimbylow et al., 2008; Vermeeren et al., 2008b; Kühn et al., 2009; Habachi et al. 2010] and the posture of the human body [Findlay and Dimbylow, 2005; Findlay et al., 2009; Uusitupa et al., 2010]. On the other 
hand, the exposure in a real environment is heterogeneous due to reflections, refractions, and transmissions of propagating waves in the environment [Saunders and Aragon-Zavala 2007].

The aim of this paper is the fast numerical assessment of the whole-body averaged SAR in a real environment for realistic human body models. Several approaches exist to estimate the whole-body averaged SAR in a real environment. These approaches can be classified in two categories according to how the exposure is modeled, i.e., deterministic or statistical. Examples of deterministic approaches are the worst-case assessment as in Kühn et al. [2009] and Vermeeren et al. [2010] and the exposure assessment in a particular area or location [Joseph et al. 2006]. In the latter the surrounding area of the measurement location is taken into account by modeling the environment for the numerical computations [Lazzi and Gandhi, 2000; Neubauer et al., 2009] or by measuring the incident fields in the environment [Gandhi and Lam 2003].

The statistical approach is more general as it aims at quantifying the absorption in a complete type of environment. In the statistical approach the incident fields or exposure in an environment are described by an appropriate statistical model. Within the statistical approach, different methods can be distinguished to assess the absorption. In [Olivier 2007] the whole-body averaged SAR $\left(\mathrm{SAR}_{\mathrm{wb}}\right)$ is determined analytically for the canonical human body model of a spheroid. Despite the fact that calculating $\mathrm{SAR}_{\mathrm{wb}}$ is analytically very fast, it cannot be used for realistic human body models. In a previous study [Vermeeren et al., 2008a], a fast numerical method has been presented to determine the whole-body absorption in a spheroid human body model in a realistic exposure environment. This method uses field distributions on a closed surface around a human body model for a limited set of incident single-plane waves to calculate the whole-body absorption for any single- or multiple-plane wave exposure. This limited set of field distributions still needs to be computed using available 3D electromagnetic solvers. The advantage of this approach is that once the limited set of basic field distributions is determined, the wholebody absorption for any realistic exposure can be determined using the linearity of Maxwell's equations. This can be performed within seconds and no time-consuming 3D electromagnetic computations are needed. Other methods encountered in literature are surrogate modeling as in [Kientega, 2011] and Monte-Carlo analysis [Iskra et al., 2011]. In contrary to the method of [Vermeeren et al., 2008a] the surrogate model presented in [Kientega, 2011] is based on only five simulations with single incident plane-wave exposure. Although, the surrogate model predicts very well the distribution of $\mathrm{SAR}_{\mathrm{wb}}$ in a real exposure environment, the relationship between the incident fields and the absorption is lost.

In the present study, the fast statistical multipath exposure (SME) method of Vermeeren et al. [2008a] for spheroid human body models is extended to heterogeneous realistic human body phantoms in realistic environments. Although the number of 3D full-wave electromagnetic computations significantly increases for realistic human body models, still a large reduction of the amount of 3D electromagnetic computations is obtained using the SME method. The advantages of this method are a significant reduction of the overall simulation time and that the relationship between the incident fields and the absorption is not lost.

First, the methodology of the fast SME method for realistic human body models is presented. Then, the method is validated, the feasibility of this fast method for realistic human bodies is discussed, and the time-consumption with respect to a completely full-wave approach is evaluated. Next, the method is applied to assess numerically $\mathrm{SAR}_{\mathrm{wb}}$ in the 6-year-old Virtual Family Boy (VFB) [Christ et al., 2008] for the Global System for Mobile Communications (GSM) downlink frequency of $950 \mathrm{MHz}$ in different environments. The compliance of the ICNIRP reference level to the basic restriction is evaluated for the heterogeneous realistic VFB and for heterogeneous exposure at $950 \mathrm{MHz}$. Finally, the results in the realistic virtual family boy are compared to the results obtained for the spheroid human body models.

\section{Materials and methods}

The method to compute quickly the whole-body SAR in a realistic human body model for heterogeneous far-field exposure is an extension of the method for spheroid human body models developed by Vermeeren et al. [2008a]. The flowchart of the method is shown in Figure 1. This flowchart is similar to the one for spheroid human body mod- 


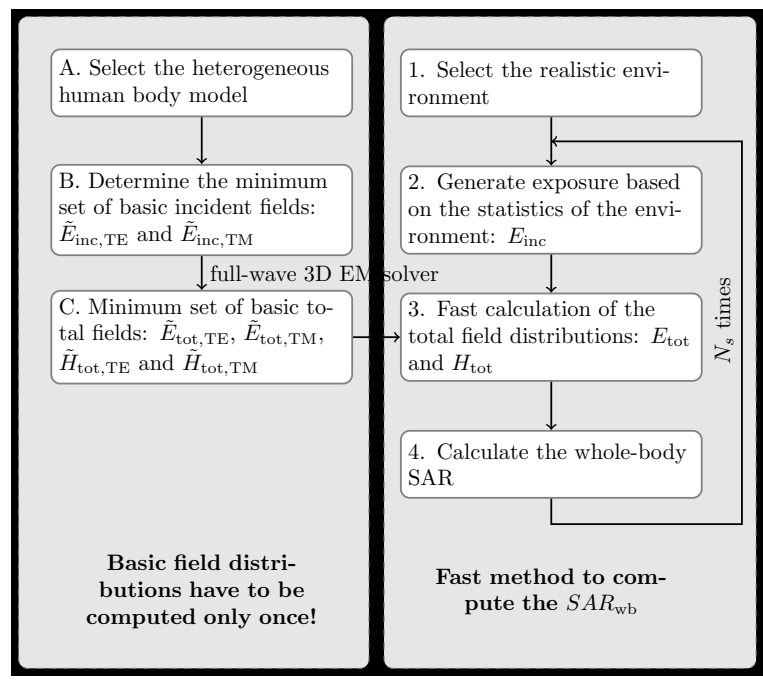

Figure 1: Flowchart of the fast method.

els. Therefore, we will not discuss into detail all the different steps, but rather focus on the steps for which the computations are different for heterogeneous realistic human body models.

\section{Statistical multipath exposure (SME) method}

The SME method consists of two major parts. The first part deals with the 3D full-wave electromagnetic simulations needed to compute the socalled basic field distributions (BFDs) for a particular human body model (Step A, B, and C of the flowchart in Figure 1). This part has to be performed only once for the selected human body model and the frequency of interest. The second part of the SME method deals with the fast calculation of the whole-body absorption for heterogeneous exposure using the pre-computed BFDs and the linearity of Maxwell's equations (Step 1, 2, 3, 4 of the flowchart).

\section{Computation of the basic field distri- butions (Step A, B, and C)}

The whole-body SAR in a body can be determined from the total electric $\left(\mathbf{E}_{\text {tot }}\right)$ and total magnetic field $\left(\mathbf{H}_{\text {tot }}\right)$ distribution on a closed surface $\mathrm{S}$ enclosing the body (see later). Assuming far-field exposure conditions (i.e., the human body in the far field of antennas and re-radiators) and no coupling between the environment and the human body, heterogeneous exposure in a realistic environment can be modeled as multiple-incident plane waves. So, if we know the total fields for all single plane waves incident on the human body from all directions, then we can quickly calculate the total fields for any heterogeneous exposure, modeled as multiple-incident plane waves, using the linearity of the Maxwell's equations. The BFDs or total fields for the singleincident plane waves have to be computed using a 3D EM solver. Fortunately, the number of BFDs to compute can be reduced in two ways. First, the fields of an incident plane wave have a certain polarization. Any polarized incident plane wave can be decomposed in two incident plane waves with mutual perpendicular polarization. These are designated as transverse electric (TE) and transverse magnetic (TM) polarized incident fields. Secondly, a plane wave can be incident upon a human body from any angle of incidence $\left(\phi_{\text {inc }}, \theta_{\text {inc }}\right)$. Obviously, it is impossible to calculate the total fields around the human body for every angle of incidence. Hence, the BFDs are computed using a 3D EM solver for a uniformly distributed set of angles of incidence $\left(\phi_{\text {inc }, m}, \theta_{\text {inc }, n}\right)$ :

$$
\begin{gathered}
\phi_{\mathrm{inc}, k}=k \Delta \phi_{\mathrm{inc}} \\
\theta_{\mathrm{inc}, l}=l \Delta \theta_{\mathrm{inc}}
\end{gathered}
$$

$\Delta \phi_{\text {inc }}$ and $\Delta \theta_{\text {inc }}$ denote the step size along azimuth and elevation, respectively. $\Delta \phi_{\text {inc }}$ and $\Delta \theta_{\text {inc }}$ depend on the number of azimuth $(\mathrm{K})$ and elevation (L) angles for which the BFDs are computed. $\Delta \phi_{\text {inc }}$ and $\Delta \theta_{\text {inc }}$ are given by:

$$
\begin{gathered}
\Delta \phi_{\text {inc }}(r a d)=\frac{2 \pi}{K} \\
\Delta \theta_{\text {inc }}(r a d)=\frac{\pi}{L-1}
\end{gathered}
$$

The number of angles of incidence for which the total fields are calculated are reduced by applying an appropriate interpolation scheme. This is possible because the total fields around the body are smooth functions of the angles of incidence. Thus, from the total set of BFDs only a limited set has to be calculated using 3D numerical techniques. These are, in what follows, referred to as 
the computed set of BFDs designated as $\tilde{\mathbf{E}}_{\text {tot,TE}}$, $\tilde{\mathbf{E}}_{\text {tot, TM }}, \tilde{\mathbf{H}}_{\text {tot,TE}}$, and $\tilde{\mathbf{H}}_{\text {tot,TM }}$ (the basic fields are designated with a tilde $(\sim)$ ).

As opposed to a spheroid human body model, a realistic human body phantom does not have any symmetry because the shape and organs of a person are not symmetric. This has two consequences. First, the basic field distributions have to be calculated for any angle of incidence, i.e., $\theta_{\text {inc }}=0 \ldots \pi$ and $\phi_{\text {inc }}=0 \ldots 2 \pi$. Secondly, the closed observation surface, where the fields are calculated, does not need to be symmetric. Hence, we have chosen a box surface around the human body phantom to reduce the size of the simulation domain in order to have a shorter overall simulation time. The electric and magnetic fields are computed in and stored for a discrete set of points on the different surfaces of the box. The points are distributed uniformly in a rectangular grid on each surface of the box. The distance between two neighboring points is a tenth of a wavelength in free space $(\lambda)$. $\lambda / 10$ is commonly used in electromagnetic theory in order to obtain accurate results. In general, the points where the fields are determined don't coincide with the grid points of the $3 \mathrm{D}$ solver. Therefore, a $2 \mathrm{D}$ interpolation has to be performed to obtain the fields in these points. Mostly the finitedifference time-domain (FDTD) method will be used for computing the basic field distributions. In an FDTD scheme the distance between the points of the grid is typically a lot smaller than $\lambda / 10$, e.g., at $950 \mathrm{MHz}$, the maximum grid step in the body is typically $2 \mathrm{~mm}$ for FDTD. Therefore, a bilinear interpolation routine is used. The routine is shown in Figure 3 for the electric field $\mathrm{E}$ in an xz-plane of the box shown in Figure 2. First, a 1D linear interpolation has been performed along the z-direction resulting in $\mathbf{E}\left(x_{k}, z_{i}\right)$ and $\mathbf{E}\left(x_{k+1}, z_{i}\right)$. Finally, a 1D linear interpolation along the x-direction renders $\mathbf{E}\left(x_{i}, z_{i}\right)$.

\section{Generation of the exposure (Step 1 and 2)}

The exposure in an environment changes in time and place. Now, let us define an exposure sample as the exposure or the incident fields on a certain time and place. In order to characterize the whole-body SAR in a human in an environment, the whole-

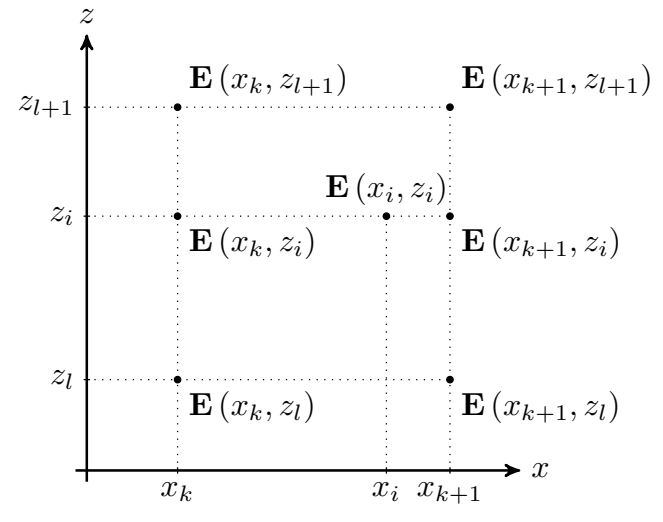

Figure 2: Bilinear interpolation in the xz-plane for the electric field.

body SAR needs to be determined for several thousands of exposure samples, i.e., at least 4000 [Vermeeren et al., 2008a], to obtain statistically relevant results. The generation of these exposure samples depends on the statistics of the environment. The generation of the exposure samples has been thoroughly discussed in Olivier [2007]. Assuming far-field exposure and no coupling between body and environment, the exposure in a realistic environment is modeled as the superposition of singleincident plane waves. The stochastic distribution of the number of incident plane waves and the parameters of the incident plane waves depend on the environment. These parameters are: amplitude $\mathrm{E}$ and phase $\alpha$ of the incident field, angles of incidence (in terms of elevation $\theta_{\text {inc }}$ and azimuth $\phi_{\text {inc }}$ ), and polarization $\psi$ of the incident field.

\section{Fast calculation of the total field dis- tribution around the human body (Step 3)}

For a realistic human body model, BFDs have to be determined for any azimuth and elevation angle $\left(\phi_{\text {inc }}, \theta_{\text {inc }}\right)$. Because the total electric and total magnetic field vary smoothly with varying angles of incidence, the BFDs only have to be simulated for a minimum set of angles of incidence. For intermediate angles of incidence, an appropriate interpolation routine is used to determine the remaining BFDs. Using an interpolation scheme reduces largely the number of computed BFDs and, thus, 


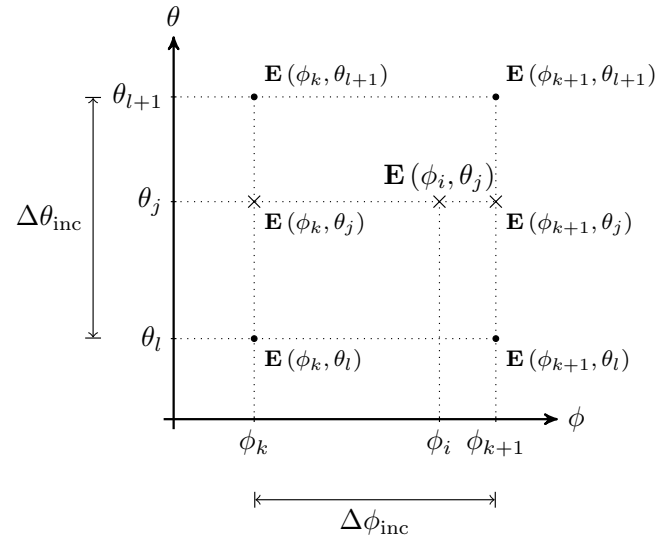

Figure 3: 2D interpolation to obtain the fields for any angle of incidence $\phi_{i}, \theta_{j}$ from the basic field distributions.

the total simulation time. The price we pay is an additional interpolation error. But, we limit this error to maximum $1 \%$ by an appropriate choice of the number of computed BFDs and interpolation scheme (see section on validation).

As opposed to rotation symmetric human body models [Vermeeren et al., 2008a], a 2D interpolation routine needs to be used for realistic human body models to find the electromagnetic fields for any angle of incidence $\left(\phi_{\text {inc }}, \theta_{\text {inc }}\right)$. The routine is shown in Figure 3. For clarity, the subscripts inc and tot are omitted in Figure 3 for angles of incidence and total fields, respectively. The total electric field $\mathrm{E}$ for the arbitrary angles of incidence $\left(\phi_{\mathrm{i}}, \theta_{\mathrm{j}}\right)$ is obtained by performing, first, a $1 \mathrm{D}$ interpolation along the elevation or $\theta$-direction resulting in $\mathbf{E}\left(\phi_{k}, \theta_{j}\right)$ and $\mathbf{E}\left(\phi_{k+1}, \theta_{j}\right)$, where $\mathrm{k}$ and $\mathrm{l}$ indicate the angles of incidence for which the BFDs are computed, and $\mathrm{i}$ and $\mathrm{j}$ denote arbitrary angles of incidence. Next, a 1D interpolation along the azimuth or $\phi$-direction results in $\mathbf{E}\left(\phi_{i}, \theta_{j}\right)$. In the section on validation two different interpolation schemes are compared: linear and cubic-spline interpolation.

\section{Calculation of $\mathrm{SAR}_{\mathrm{wb}}($ Step 4)}

According to IEEE C95.3-2002 [IEEE SCC28, 2002] the whole-body SAR is averaged over the mass of the human body model and is given by:

$$
S A R_{\mathrm{wb}}=\frac{1}{M} \int_{R} S A R(\mathbf{r}) d m
$$

with $\mathrm{R}$ the region over which the SAR is averaged over the mass $\mathrm{M}$ of the human body. The massaveraged whole-body SAR is equal to the ratio of the absorbed power in the body (Pabs) and the $\operatorname{mass}(\mathrm{M})$ :

$$
S A R_{\mathrm{wb}}=\frac{P_{\mathrm{abs}}}{M}
$$

Thus, the absorbed power can be determined from the nett power flux through a closed surface $(\mathrm{S})$ around the body by integrating the Poynting vector $(\wp)$ over the closed surface and taking the real part of it:

$$
P_{\mathrm{abs}}=\Re\left\{\oint_{R} \wp \cdot d s\right\}
$$

The Poynting vector in every point of the closed surface is determined from the total electric $\left(\mathbf{E}_{\text {tot }}\right)$ and the total magnetic field vector $\left(\mathbf{H}_{\text {tot }}\right)$. The total fields are the superposition of the incident fields $\left(\mathbf{E}_{\text {inc }}, \mathbf{H}_{\text {inc }}\right)$ and the scattered fields $\left(\mathbf{E}_{\text {scat }}, \mathbf{H}_{\text {scat }}\right)$ at the surface of the body. Thus, the fast calculation of $\mathrm{SAR}_{\mathrm{wb}}$ is based on the linearity of Maxwell's equations, on the knowledge of the total fields on a closed surface around the human body for a limited set of single-incident plane waves, and on an appropriate choice of interpolation schemes.

\section{Validation of the method}

The presented statistical multipath exposure method (SME) is a very fast alternative for the time-consuming computations of a 3D EM solver, designated as FW (full wave), once the basic field distributions are available. Because the SME method serves as a substitute for a 3D EM solver, one has to validate this method with the results obtained by the 3D EM solver. The quantities calculated with the SME method and a full-wave 3D EM solver has as subscript SME and FW, respectively. Therefore, we define the relative error on $\mathrm{SAR}_{\mathrm{wb}}$ as follows:

$$
e r r_{\mathrm{rel}, S A R_{\mathrm{wb}}}=100 \frac{S A R_{\mathrm{wb}, \mathrm{SME}}-S A R_{\mathrm{wb}, \mathrm{FW}}}{S A R_{\mathrm{wb}, \mathrm{FW}}}
$$


Validating the method does not only give us an idea about the uncertainty, but will also give us an idea about the number of basic field distributions which need to be computed using a full-wave numerical tool. Or, in other words, the validation will also determine the optimum settings for the spacing between the angles of incidence $\left(\Delta \phi_{\text {inc }}, \Delta \theta_{\text {inc }}\right)$ for which the BFDs are computed. Furthermore, we will also look how the interpolation scheme, linear or cubic spline, influences the uncertainty, and the spacing $\left(\Delta \phi_{\text {inc }}, \Delta \theta_{\text {inc }}\right)$ when determining the fields for an arbitrary angle of incidence.

The validation of the fast SME method is performed for multi-path exposure. Therefore, 100 samples of GSM downlink exposure at $950 \mathrm{MHz}$ in an urban-macrocell environment are generated using the statistical method of Olivier [2007]. The 6-year-old Virtual Family Boy (VFB) [Christ et al., 2008] is selected as human body model. The VFB consists of 81 different tissues. The tissues of the VFB are mapped to the tissues available in the Gabriel database [Gabriel et al., 1996] for which the dielectric properties have been determined experimentally. The whole-body SAR in VFB for the generated 100 exposure samples is calculated using the full-wave 3D FDTD solver SEMCAD-X (v14.0, SPEAG, Zürich, Switzerland) and the SME method.

As mentioned before, simulating the BFDs for all angles of incidence is impossible. Hence, the BFDs are only simulated for a limited set of angles of incidence. The BFDs for intermediate angles of incidence are determined by interpolation because the total fields around the human body are smooth. The 2D-interpolation in $\left(\Delta \phi_{\text {inc }}, \Delta \theta_{\text {inc }}\right)$-plane is achieved by performing two 1D-interpolations. This paragraph discusses the difference between two well-known interpolation schemes: linear and cubic spline. The spacing between the angles of incidence are fixed to $10^{\circ}$ for azimuth angles $\left(\Delta \phi_{\text {inc }}=10^{\circ}\right)$ and $5^{\circ}$ for elevation angles $\left(\Delta \theta_{\text {inc }}=5^{\circ}\right)$. Figure 4 shows the errors on $\mathrm{SAR}_{\mathrm{wb}}$ for linear and cubic-spline interpolation of the BFDs, respectively. An excellent agreement has been observed between the SME calculations and the SEMCAD-X calculations for the cubic-spline interpolation as the mean $(\mu)$ and the standard deviation $(\sigma)$ of the relative error on $\mathrm{SAR}_{\mathrm{wb}}$ in the human body is $-0.3 \%$ and $0.4 \%$, respectively (see Figure 4). A linear interpolation scheme underesti-

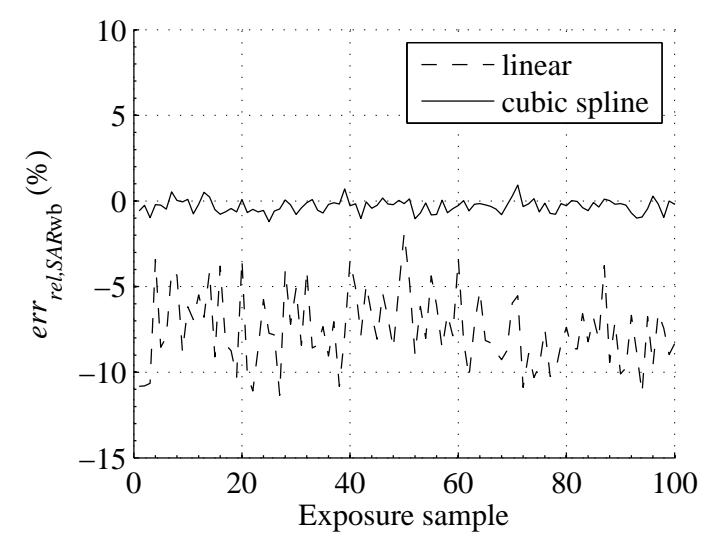

Figure 4: Relative error in the $\mathrm{SAR}_{\mathrm{wb}}$ in the Virtual Family boy (VFB) exposed to EMF from GSM downlink at $950 \mathrm{MHz}$ in an urban-macrocell environment for linear and cubic-spline interpolation.

mates $\mathrm{SAR}_{\mathrm{wb}}$ by $-7.5 \%$ with a standard deviation of $2.2 \%$, respectively (see Figure 4 ). A cubic-spline scheme estimates better the total fields around the human body than the linear scheme for the same number of angles of incidence. The cost of a cubicspline interpolation is approximately a doubling of the computing time of the BFDs with respect to a linear interpolation scheme. Nevertheless, the cubic-spline interpolation is preferred because it reduces significantly the number of computed BFDs which have to be determined by a full-wave $3 \mathrm{D}$ EM solver. The cubic-spline interpolation scheme is used from this point on unless otherwise stated.

The number of BFDs which have to be calculated by $3 \mathrm{D}$ numerical means is mainly determined by the performance of the interpolation scheme for the angles of incidence $\left(\Delta \phi_{\text {inc }}, \Delta \theta_{\text {inc }}\right)$. The better the interpolation scheme the larger the spacing between the angles of incidence, and the less BFDs to calculate. In the previous paragraph it is observed that the cubic-spline interpolation is preferable over a linear interpolation. Now, the influence of the spacing $\Delta \phi_{\text {inc }}$ and $\Delta \theta_{\text {inc }}$ on $\operatorname{err}_{\mathrm{rel}, S A R_{\mathrm{wb}}}$ is investigated. The spacing along azimuth is varied between $10^{\circ}$ and $40^{\circ}$ in steps of $10^{\circ}$. The spacing along elevation is varied between $5^{\circ}$ and $20^{\circ}$ in steps of $5^{\circ}$. The mean and standard deviation of $\operatorname{err}_{\mathrm{rel}, S A R_{\mathrm{wb}}}$ are shown in Figure 5. As expected, the smallest error is found for the smallest spacing, i.e., $\Delta \phi_{\text {inc }}=10^{\circ}$ and $\Delta \theta_{\text {inc }}=5^{\circ}$. The mean of $\operatorname{err}_{\mathrm{rel}, S A R_{\mathrm{wb}}}$ varies 
between $0.4 \%\left(\Delta \phi_{\text {inc }}=10^{\circ}, \Delta \theta_{\text {inc }}=5^{\circ}\right)$ and $23 \%$ for $\left(\Delta \phi_{\text {inc }}=40^{\circ}, \Delta \theta_{\text {inc }}=20^{\circ}\right)$. Also the standard variation increases with increasing spacing between the azimuth and elevation angles. The variation of $\operatorname{err}_{\mathrm{rel}, S A R_{\mathrm{wb}}}$ along the elevation angle is larger than along the azimuth angle.

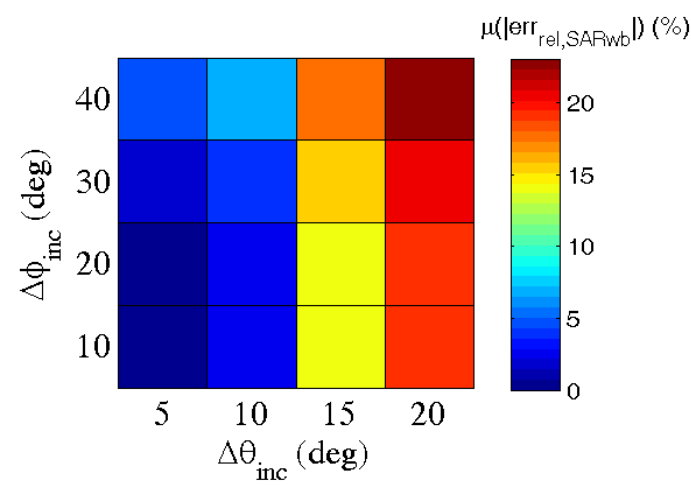

(a)

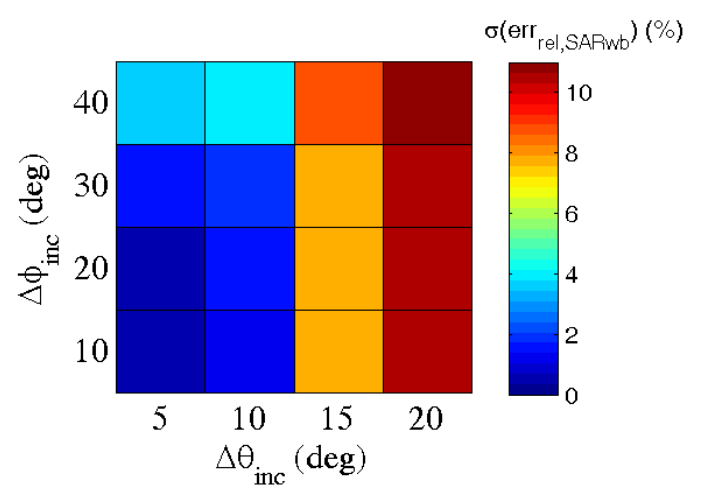

(b)

Figure 5: (a) Mean and (b) standard deviation of the relative error on the absorption in the VFB exposed to EMF from GSM downlink at $950 \mathrm{MHz}$ in an urban-macrocell environment for varying number of BFDs.

\section{Uncertainty assessment}

The previous section validated the SME calculations with respect to full-wave numerical computations. The error can be less than $1 \%$ depending on the number of available BFDs and the interpolation routines. To assess the overall uncertainty [Taylor and Kuyatt, 1994] we also have to take into account the uncertainties introduced by the 3D EM solver used for the computations of the BFDs and the uncertainties on the dielectric properties and mass densities of the body tissues. In this study the FDTD method available in SEMCAD-X (v14.0, Speag, Zürich, Switzerland) is applied to compute the BFDs. A comprehensive uncertainty analysis of the FDTD calculations is outside the scope of the present study. Hence, we assessed the uncertainty for the BFD calculations based on previous studies [Findlay and Dimbylow, 2006; Kühn et al., 2009; Bakker et al., 2010; Bakker et al., 2011]. The expanded uncertainty $\mathrm{U}(\mathrm{k}=2)$ on $\mathrm{SAR}_{\mathrm{wb}}$ in a heterogeneous human body model exposed to an single-incident plane wave equals $21.2 \%$ using the FDTD method according to Bakker et al. [2010, 2011]. This large uncertainty is mainly introduced by the uncertainty on the dielectric properties and mass density of the tissues. Assuming that the error of the SME method with respect to the fullwave FDTD calculations is not correlated with the FDTD uncertainties and taking into account an additional error of $1 \%$ for the SME calculations, the expanded uncertainty $\mathrm{U}(\mathrm{k}=2)$ remains $21.2 \%$. The additional error introduced by the fast SME calculations is negligible with respect to the uncertainty of $21.2 \%$ for the FDTD computations. Table 1 lists the standard uncertainties involved in the SME method.

\section{Results}

The methodology of the fast method for a realistic human body phantom is applied for the Virtual Family Boy (VFB) [Christ et al., 2008]. It has been shown by Vermeeren et al. [2008b], Kühn et al. [2009], and Uusitupa et al. [2010] that the wholebody absorption increases for smaller human body models. Again, the tissues of the VFB are mapped to the tissues available in the Gabriel database [Gabriel et al., 1996]. The 6-year-old VFB has been exposed to radio-frequency electromagnetic fields at the GSM downlink frequency of $950 \mathrm{MHz}$ in an urban-macrocell environment [Olivier, 2007].

\section{Time consumption}

The FDTD computations were done on a $2.2 \mathrm{GHz}$ dual-core workstation (Precision 690, Dell, Round Rock, Texas, USA) with Graphics Processing Unit 
Table 1: The standard combined uncertainty uc and the expanded uncertainty $\mathrm{U}$ on $\mathrm{SAR}_{\mathrm{wb}}$ for the SME method applied to realistic human body models based on FDTD simulations.

\begin{tabular}{lc}
\hline & SAR $_{\mathrm{wb}}$ Std unc. (\%) \\
\hline \hline Discretization & \\
(a) spatial resolution & 2.5 \\
(b) non-uniform grid & 0.5 \\
\hline ABC + & 3.5 \\
steady state & \\
deviation & \\
\hline Wave homogeneity & \\
\hline$\rho^{1,2}$ & 1.0 \\
\hline$\epsilon_{\mathrm{r}}, \sigma^{1,2}$ & 5.0 \\
(a) Age-dependence & \\
(b) Tissue assignment & 5.8 \\
(c) 4-Cole-Cole & 5.4 \\
(d) Post-mortal changes & 1.5 \\
\hline SME method & 1.5 \\
\hline \hline $\mathrm{u}_{\mathrm{c}}(\mathrm{k}=1)$ & 1 \\
\hline U $(\mathrm{k}=2)$ & 10.6 \\
\hline 1 Bakker at al. 2010 & 21.2 \\
2 Bakker at al. 2011 &
\end{tabular}

(GPU) computing (NVIDIA Quadro Plex 2200 Model D2, NVIDIA, Santa Clara, California, USA) and $32 \mathrm{~GB}$ of RAM. The calculations for the SME method were performed on a $3 \mathrm{GHz}$ quad-core PC with $4 \mathrm{~GB}$ of RAM (Optiplex 760, Dell, Round Rock, Texas, USA). Computing the BFDs took 9.3 days. Once the BFDs are available, assessing $\mathrm{SAR}_{\mathrm{wb}}$ in a single environment (i.e., $5000 \mathrm{ex}-$ posure samples) took only $8 \mathrm{~h}$, whereas assessing $\mathrm{SAR}_{\mathrm{wb}}$ with only full-wave computations should take 17.4 days. So, using the SME method is $45 \%$ faster than using solely full-wave calculations (for 5000 exposure samples). Moreover, the gain in time increases when more environments are considered as the BFDs have to be calculated only once!

The whole-body averaged SAR in the Virtual Family Boy in a realistic environment for the GSM downlink frequency

The cumulative distribution function (cdf) of the whole-body averaged SAR in the Virtual Family
Boy for four realistic exposure environments, i.e., urban-macrocell, urban-microcell, outdoor-indoor, and indoor-picocell for the GSM downlink frequency of $950 \mathrm{MHz}$ is shown in Figure 6. The incident power density $\left(S_{\text {inc }}\right)$ for all the exposure samples equals the ICNIRP reference level (RL). For general public exposure, RL equals $4.75 \mathrm{~W} / \mathrm{m}^{2}$ at $950 \mathrm{MHz}$. In order to verify easily the compliance of $\mathrm{SAR}_{\mathrm{wb}}$ with basic restrictions, the following ratio is defined:

$$
R_{\mathrm{wb}}=\frac{\left.S A R_{\mathrm{wb}}\right|_{S_{\mathrm{inc}}=R L}}{B R_{\mathrm{wb}}}
$$

with $\mathrm{BR}_{\mathrm{wb}}$ the basic restriction for the whole-body SAR and for the corresponding reference level. Figure 6 shows the cdf for $R_{w b}$ for the investigated configurations. In every environment the wholebody SAR is calculated for 5000 multi-path exposure samples in order to obtain relevant statistics in every environment. The 50th ( $\left.\mathrm{p}_{50}\right)$, 90th ( $\left.\mathrm{p}_{90}\right)$, 95th ( $\left.\mathrm{p}_{95}\right)$, and 99th ( $\left.\mathrm{p}_{99}\right)$ percentile of $\mathrm{SAR}_{\mathrm{wb}}$ and $\mathrm{R}_{\mathrm{wb}}$ in the VFB in the different environments are shown in Figure 7. It is observed that the reference level is not always compliant with the whole-body basic restriction, but it is unlikely that the basic restriction will be exceeded for an incident power density equal to the reference level as $\mathrm{SAR}_{\mathrm{wb}}$ exceeds $\mathrm{BR}_{\mathrm{wb}}$ in only $0.26 \%$ of the exposure samples (averaged over the different investigated environments). The median of $R_{\mathrm{wb}}$ or $\mathrm{p}_{50}\left(\mathrm{SAR}_{\mathrm{wb}}\right)$ is about $2.5 \mathrm{~dB}$ lower than the $\mathrm{BR}_{\mathrm{wb}}$.

The whole-body SAR for single plane-wave exposure is also shown in Figure 6 and it is indicated by markers. Only frontal incidence in a horizontal plane is considered as this yields the worst-case single plane-wave exposure in heterogeneous human body models according to Kühn et al. [2009]. Two different polarizations are considered, i.e., vertical or E-polarization, and horizontal or H-polarization. The worst-case single incident plane wave for the VFB is E-polarized and induces an $\mathrm{SAR}_{\mathrm{wb}}$ value which is $1.3 \mathrm{~dB}$ below the basic restriction. Averaged over the four considered environments, $3.6 \%$ of the multi-path exposure samples (or, heterogeneous exposure) have a higher $\mathrm{SAR}_{\mathrm{wb}}$ than for worst-case single-incident plane-wave exposure (or, homogeneous exposure).

The cdf of $\mathrm{SAR}_{\mathrm{wb}}$ does not differ significantly between the considered environments for a constant incident power density as shown in Figure 6 and 


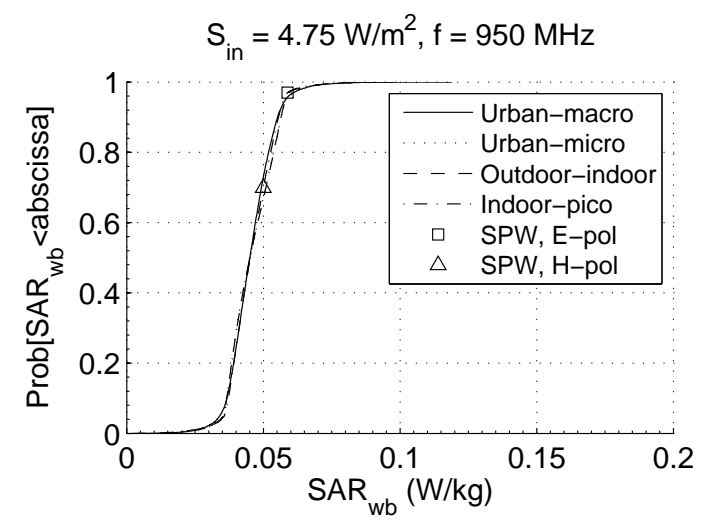

(a)

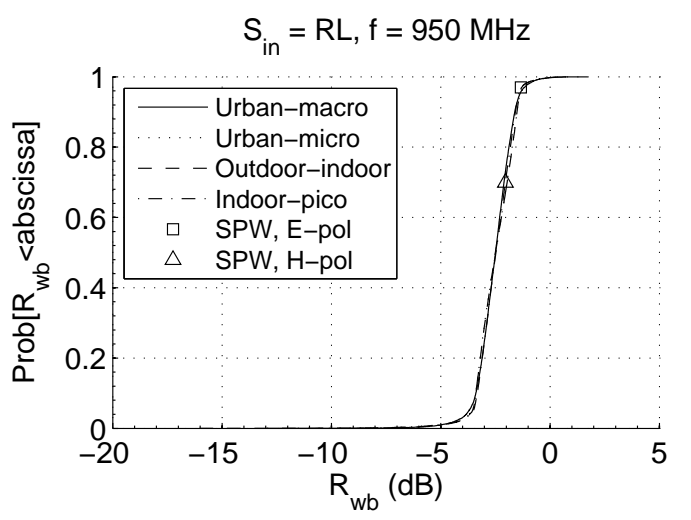

(b)

Figure 6: Cumulative distribution function of (a) $\mathrm{SAR}_{\mathrm{wb}}$ and (b) $\mathrm{R}_{\mathrm{wb}}$ in the VFB in four realistic environments exposed to EMF from GSM downlink at $950 \mathrm{MHz}$. Incident power density equaled the ICNIRP reference level for general public exposure, that is, $4.75 \mathrm{~W} / \mathrm{m}^{2}$ at $950 \mathrm{MHz}$. The results for an $\mathrm{E}$ - and $\mathrm{H}$-polarized frontal incident plane wave are indicated by markers; SPW = single plane wave.

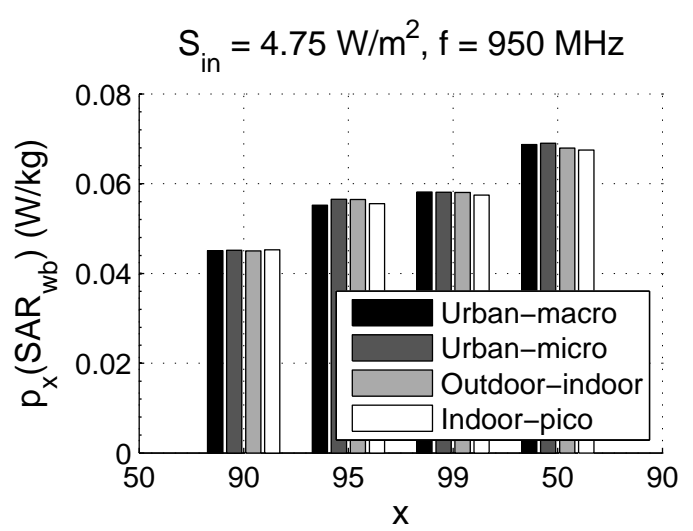

(a)

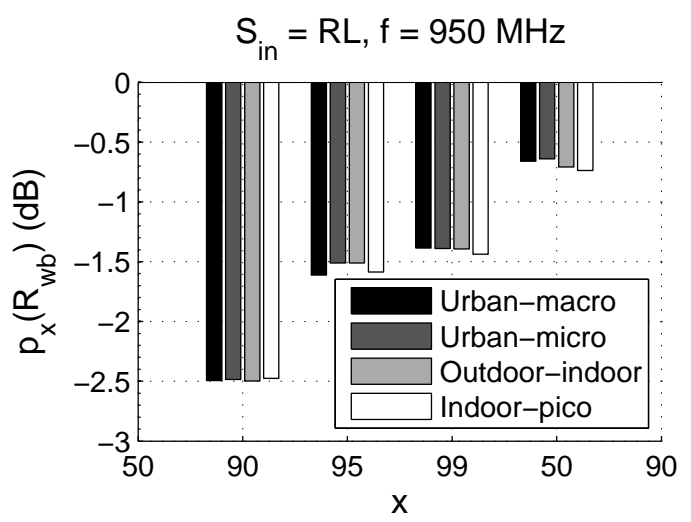

(b)

Figure 7: 50th, 90th, 95th, and 99th percentile of (a) $\mathrm{SAR}_{\mathrm{wb}}$ and (b) $R_{\mathrm{wb}}$ in the VFB exposed to EMF from GSM downlink at $950 \mathrm{MHz}$ in different environments. Incident power density equaled the ICNIRP reference level for general public exposure. 


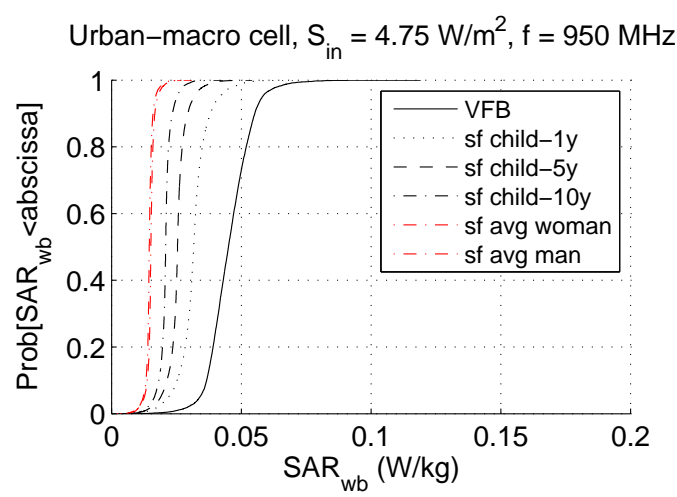

(a)

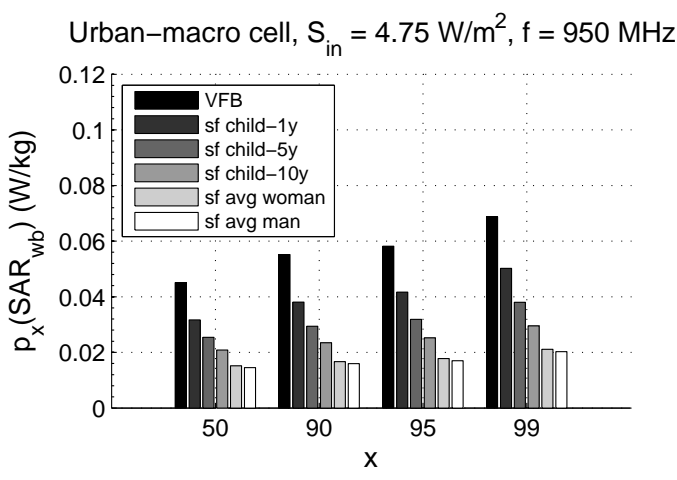

(b)

Figure 8: (a) The cdf of $\mathrm{SAR}_{\mathrm{wb}}$ and (b) the 50th-, 90th-, 95th-, and 99th-percentile of $\mathrm{SAR}_{\mathrm{wb}}$ in VFB and SF human body models in an urban-macro cell environment exposed to EMF from GSM downlink at $950 \mathrm{MHz}$. The incident power density equaled the ICNIRP reference level for general public exposure, i.e. $4.75 \mathrm{~W} / \mathrm{m}^{2}$ at $950 \mathrm{MHz}$.

Figure 7. The maximum of $\mathrm{p}_{50}, \mathrm{p}_{90}, \mathrm{p}_{95}$, and $\mathrm{p}_{99}$ is $0.5 \%, 2.4 \%, 1.2 \%$, and $2.2 \%$ larger than the minimum percentiles, respectively.

Comparison of the whole-body SAR for the heterogeneous VFB and the spheroid human body model in a realistic exposure environment at $950 \mathrm{MHz}$

In Vermeeren et al. [2008b] the whole-body SAR in spheroid human body models in realistic exposure environments is investigated for frequencies ranging from $150 \mathrm{MHz}$ to $950 \mathrm{MHz}$. In this section the whole-body SAR in the realistic human body model VFB is compared to the whole-body SAR obtained in spheroid models. The dimensions of the spheroids are taken from Durney et al. [1986]. The homogeneous spheroid has been assigned the dielectric properties of average head as suggested by the International Electrotechnical Commission (IEC) in IEC62209 [IEC, 2005]. Because the statistics of $\mathrm{SAR}_{\mathrm{wb}}$ do not show a significant difference between different environments, in what follows only the urban-macro cell environment is considered. Figure 8 shows the cdf of the whole-body SAR for the VFB and the spheroid human body models in an urban-macro cell environment for the GSM downlink frequency of $950 \mathrm{MHz}$. It is clearly observed that the spheroid homogeneous human body models underestimate the whole-body SAR in the realistic heterogeneous VFB model. The median or $\mathrm{p}_{50}$ of $\mathrm{SAR}_{\mathrm{wb}}$ for the 5-year-old spheroid model is $2.5 \mathrm{~dB}$ lower than for the 6-year-old VFB (see Figure 8). Because the exposure for the various models is identical, the difference in $\mathrm{SAR}_{\mathrm{wb}}$ is caused by a difference in morphology of the human body models. The increase in $\mathrm{SAR}_{\mathrm{wb}}$ in the VFB cannot be completely attributed to the difference in weight and size of the models because $\mathrm{SAR}_{\mathrm{wb}}$ in the VFB is larger than for all the spheroid human body models. Hence, the tissue properties as well as the shape of the model play an important role in the difference of $\mathrm{SAR}_{\mathrm{wb}}$. To investigate the influence of the tissue properties and the shape of the models, the whole-body SAR for single incident plane-wave exposure is determined in the heterogeneous and homogeneous VFB, and the homogeneous 1-yearold spheroid child. For the homogeneous VFB, the dielectric properties of all the tissues and air cavities are assigned the same value as for the spheroid models. Figure 9 shows $\mathrm{SAR}_{\mathrm{wb}}$ for different elevation angles of the incident plane wave and an incident field strength of $1 \mathrm{~V} / \mathrm{m}$. Vertical (E) and horizontal polarization $(\mathrm{H})$ are considered. Assigning a homogeneous tissue to the VFB reduces the whole-body absorption. The difference between the homogeneous VFB and the homogeneous 1-yearold spheroid child is due to the difference in mass and shape of the body models. So, the heterogeneous tissues and the irregular shape of realistic human body models increases the whole-body averaged SAR compared to homogeneous spheroid 
Table 2: The standard deviation of $\mathrm{SAR}_{\mathrm{wb}}$ in VFB and SF human body models in an urban-macro cell environment exposed to electromagnetic fields from GSM downlink at $950 \mathrm{MHz}$. The incident power density equaled the ICNIRP reference level for general public exposure, i.e. $4.75 \mathrm{~W} / \mathrm{m}^{2}$ at $950 \mathrm{MHz}$.

\begin{tabular}{lc}
\hline Human body model & $\sigma\left(\mathrm{SAR}_{\mathrm{wb}}\right)(\mathrm{mW} / \mathrm{kg})$ \\
\hline \hline Spheroid 1-year old & 1.6 \\
\hline Spheroid 5-year old & 1.8 \\
\hline Spheroid 10-year old & 2.7 \\
\hline Spheroid average woman & 4.0 \\
\hline Spheroid average man & 5.9 \\
\hline VFB & 8.2 \\
\hline
\end{tabular}

human body models.

Figure 8 also shows that the variability of the $\mathrm{SAR}_{\mathrm{wb}}$ around the mean is the largest in the realistic VFB human body model. Table 2 lists the standard deviation $(\sigma)$ of $\mathrm{SAR}_{\mathrm{wb}}$ in the different human body models for an urban-macro cell environment. For the spheroid human body models, $\sigma$ increases with size of the spheroids. The highest $\sigma$ is observed for the realistic VFB model. The larger variability of $\mathrm{SAR}_{\mathrm{wb}}$ is caused by the irregular shape of the VFB. This can be partly explained from Figure 9 . Figure 9 shows the variation of $\mathrm{SAR}_{\mathrm{wb}}$ along the elevation direction (E- and H-polarization) for single incident plane wave exposure. The variation of $\mathrm{SAR}_{\mathrm{wb}}$ is larger for the irregular-shaped, realistic human body model of the VFB. The heterogeneity of the tissues has a minor effect on the variation of $\mathrm{SAR}_{\mathrm{wb}}$. Moreover, the spheroid human body models do not show a variation of $\mathrm{SAR}_{\mathrm{wb}}$ along the azimuth direction because of the rotation symmetry. Due to the absence of rotation symmetry in the $\mathrm{VFB}, \mathrm{SAR}_{\mathrm{wb}}$ in the $\mathrm{VFB}$ varies also along the azimuth direction.

\section{Discussion}

In this study a fast numerical SME method is presented for the assessment of the whole-body averaged SAR induced in heterogeneous human body models in a multi-path environment. Assessing the whole-body SAR in a real environment requires a statistical approach [Vermeeren et al., 2008a]. Different approaches exist. In specific cases, determin-

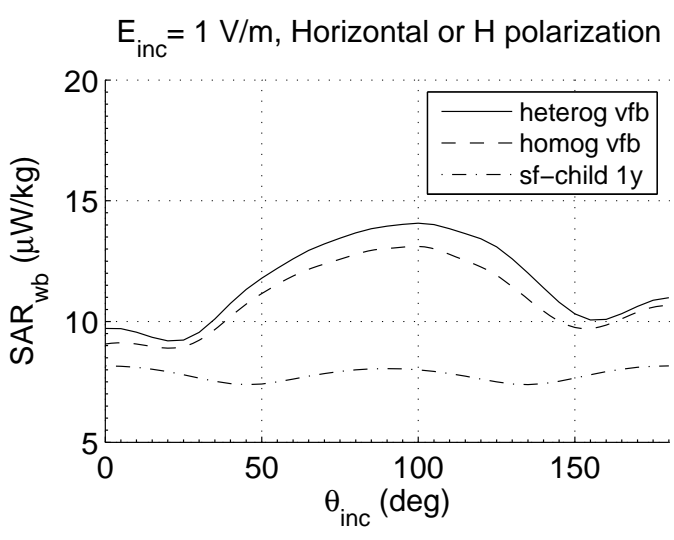

(a)

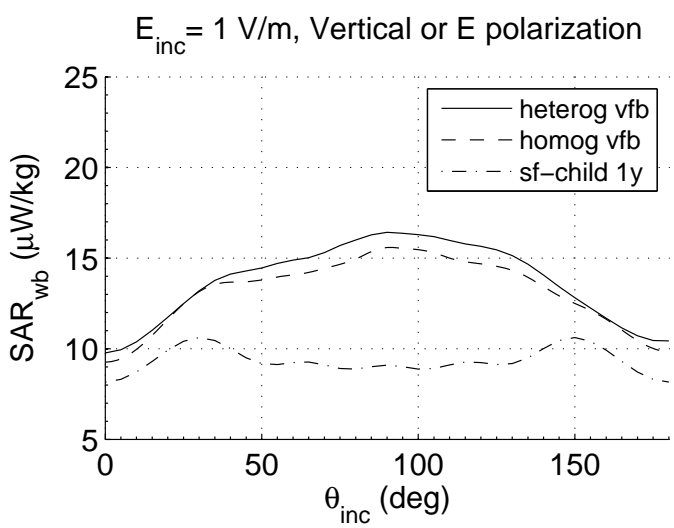

(b)

Figure 9: The whole-body SAR in the heterogeneous and homogeneous VFB, and the 1-year-old spheroid human body model for (a) vertical and (b) horizontal polarized single incident plane-wave exposure at $950 \mathrm{MHz}$ with varying elevation angle.

istic methods using, e.g., 3D ray-tracing tools are applied [Neubauer et al., 2009]. For more generic environments such as urban and indoor-outdoor environments, surrogate modeling, Monte-Carlo analysis, and the proposed statistical multi-path exposure method (SME method) can be applied. With respect to the surrogate modeling and the MonteCarlo method, the SME method keeps the relation between the incident fields and the induced wholebody SAR. The price for this relationship is a larger set of $3 \mathrm{D}$ numerical computations. Keeping the relationship between the incident fields and the induced SAR allows us to investigate in the future 
which exposures causes high induced SAR.

Despite the increased number of full-wave computations with respect to surrogate modeling and Monte-Carlo simulations, still a reduction in simulation time (with a factor 2 or more) is obtained with the presented approach with respect to a complete full-wave analysis. The SME method reduces largely the computational complexity once the BFDs are known compared to a solely fullwave approach. The exposure in a multi-path environment is modeled as the superposition of several single-incident plane waves. We applied the FDTD method to compute the BFDs. In the FDTD method a total-field scattered-field (TFSF) method is used to model a single-incident plane wave. In order to model multi-path exposure in an FDTD solver, several plane-wave sources are placed in the simulation domain whereas in the SME method the total fields on the closed surface around the body model for multi-path exposure are obtained by superposition of the total fields of the individual single-incident plane waves. In addition, also the data is significantly reduced with respect to a full-wave approach as only the electric and magnetic fields on the closed surface around the human body model are necessary to calculate the wholebody SAR. In an FDTD scheme the fields need to be computed in every cell of the simulation domain resulting in a large overhead of data.

The Statistical Multipath Exposure (SME) method for realistic human body models is an extension of the SME method for rotation and mirror (in a horizontal plane) symmetric human body models such as a spheroid. Due to the absence of the rotation and mirror symmetry the number of computed basic field distributions (BFDs) increases enormously. Despite this increase in the amount of full-wave 3D electromagnetic simulations for computing the BFDs, the proposed extension of the SME method still shows a large reduction in overall simulation time with respect to a completely full-wave numerical approach. The method has been improved to deal efficiently with the increased amount of data with respect of the SME method for rotation symmetric bodies: for every incident plane wave in the set of incident multiple plane waves, only the neighboring BFDs are loaded instead of loading all the BFDs in memory at the beginning of the algorithm. Another difference is the closed surface around the human body. For re- alistic human body models a closed boxed surface is sufficient. Consequently, only a $2 \mathrm{D}$ interpolation scheme is sufficient instead of the 3D interpolation for rotation symmetric human body models. A final significant difference between realistic and rotation symmetric human body models is that the BFDs also have to be computed for varying azimuth angle. This is not the case for the rotation symmetric human body models because the fields for a different azimuth angle can be determined by a rotation of the fields from another azimuth angle. This also implies that for realistic human body models a 2D interpolation in the azimuth-elevation plane instead of the 1D interpolation for rotation symmetric human body models.

The uncertainties of the SME method are due to the full-wave computations for the BFDs, the large uncertainty on the dielectric properties and the mass density of the human body tissues, and the algorithms used to assess $\mathrm{SAR}_{\mathrm{wb}}$ for multi-path exposure (referred to as SME calculations). As discussed in the validation, errors of less than $1 \%$ can be obtained with respect to the full-wave computations. Thus, the uncertainty is mainly determined by the uncertainty of the full-wave tool. Uncertainty of FDTD computations is studied thoroughly in Bakker et al. [2010]. The overall uncertainty is here estimated to be equal to $21 \%$.

The fast SME method is applied to the VFB in four realistic environments for the GSM downlink frequency at $950 \mathrm{MHz}$. This study confirms the findings of Vermeeren et al. [2008], Kühn et al. [2009], Kientega et al. [2011] that multiple planewave exposure can induce a higher whole-body SAR than the worst-case single incident plane-wave exposure. The influence of the morphology was also investigated. We showed that internal and external morphology largely influences the whole-body SAR. Our findings agree with Conil et al. [2008], Habachi et al. [2010]. The increase in whole-body SAR in heterogeneous tissue distribution with respect to a homogeneous tissue distribution can be attributed to the difference in tissue properties and standing wave effects in tissues with low water content [Christ et al., 2006]. The influence of posture on $\mathrm{SAR}_{\mathrm{wb}}$ was not investigated, but based on Uusitupa et al. [2010] it is expected that the variation of $\mathrm{SAR}_{\mathrm{wb}}$ will increase when taking into account different postures of the human body model. We also showed that at higher frequencies the influence 
of the environment on the statistics of the wholebody SAR is negligible. Finally, this paper also clearly confirms that the deduction of the ICNIRP reference levels based on the worst-case homogeneous exposure for homogeneous human body models does not yield a conservative approach. In addition, Kühn et al. [2009], Uusitupa et al. [2010], and Bakker et al. [2010, 2011] also showed that for worst-case single incident plane-wave exposure and multi-path exposure taking one reflection into account, the basic restrictions might be violated at other frequencies by larger amounts than at $950 \mathrm{MHz}$, especially at frequencies around $2 \mathrm{GHz}$. This is mainly attributed to the relaxation of the reference levels at frequencies above $2 \mathrm{GHz}$.

The current SME method only applies for assessing the whole-body SAR because the absorption is determined from the electromagnetic fields on a closed surface around the body. To calculate the organ-specific and localized SAR requires the knowledge of the fields inside the human body. Extending the SME method for organ-specific and localized SAR is currently under investigation.

\section{Conclusions}

The statistical multi-path exposure tool is extended so that it can be used for heterogeneous realistic human body models. The numerical method is investigated thoroughly for the Virtual Family Boy exposed to the GSM downlink frequency of $950 \mathrm{MHz}$ in several realistic environments. The method is a fast alternative for full-wave 3D electromagnetic solvers to assess numerically the absorption in a body. At $950 \mathrm{MHz}$, the gain in time can be as high as a factor 2 or more. The exposure samples can not only be generated by the statistical method of Olivier [2007], but can also be exported from a 3D ray tracing tool. The whole-body absorption in the Virtual Family Boy for the GSM downlink at $950 \mathrm{MHz}$ has been investigated for several realistic environments. It is shown that the whole-body SAR exceeds the basic restrictions for an incident power density equal to the ICNIRP reference level for $0.3 \%$ of the exposure samples. The whole-body SAR for realistic exposure also exceeds for $3.6 \%$ of the exposure samples the whole-body SAR in for worst-case single plane-wave exposure. Furthermore, it is also shown that the whole-body
SAR in homogeneous spheroid human body models and for realistic multi-path exposure underestimates the whole-body SAR in heterogeneous realistic human body models.

\section{Acknowledgments}

W. Joseph is a Post-Doctoral Fellow of the FWO-V (Research Foundation-Flanders).

\section{References}

Bakker JF, Paulides MM, Christ A, Kuster N, van Rhoon GC. 2010. Assessment of induced SAR in children exposed to electromagnetic plane waves between $10 \mathrm{MHz}$ and $5.6 \mathrm{GHz}$. Phys Med Biol 55:3115-3130.

Bakker JF, Paulides MM, Christ A, Kuster N, van Rhoon GC. 2011. Corrigendum for Assessment of induced SAR in children exposed to electromagnetic plane waves between $10 \mathrm{MHz}$ and 5.6 GHz. Phys Med Biol 56:2883.

Christ A, Klingenböck A, Samaras T, Goiceanu C, Kuster N. 2006. The dependence of electromagnetic far-field absorption on body tissue composition in the frequency range from 300 $\mathrm{MHz}$ to $6 \mathrm{GHz}$. IEEE Trans Microw Theory Tech 54:2188-2195.

Christ A, Kainz W, Hahn EG, Honegger K, Zefferer M, Neufeld E, Rascher W, Janka R, Bautz W, Chen J, Kiefer B, Schmitt P, Hollenbach HP, Shen J, Oberle M, Szczerba D, Kam A, Guag JW, Kuster N. 2010. The Virtual Family - development of surface-based anatomical models of two adults and two children for dosimetric simulations. Phys Med Biol 48:N23-N38.

Conil E, Hadjem A, Lacroux F, Wong MF, Wiart J. 2008. Variability analysis of SAR from 20 $\mathrm{MHz}$ to $2.4 \mathrm{GHz}$ for different adult and child models using finite-difference time-domain. Phys Med Biol 53:1511-1525.

Dimbylow PJ, Hirata A, Nagaoka T. 2008. Intercomparison of whole-body averaged SAR in European and Japanese voxel phantoms. Phys Med Biol 53:5883-5897. 
Durney CH, Massoudi H, Iskander, MF. 1986. Radiofrequency Radiation Dosimetry Handbook: 4th ed. USAF School of Aerospace Medicine, Brooks Air Force Base, Texas, USA. Findlay RP, Dimbylow PJ. 2006. Variations in calculated SAR with distance to the perfectly matched layer boundary for a human voxel model. Phys Med Biol 51-N411-N415.

Findlay RP, Dimbylow PJ. 2005. Effects of posture on FDTD calculations of specific absorption rate in a voxel model of the human body. Phys Med Biol 50:3825-3835.

Findlay RP, Lee AK, Dimbylow PJ. 2009. FDTD calculations of SAR for child voxel models in different postures between $10 \mathrm{MHz}$ and $3 \mathrm{GHz}$. Radiat Prot Dosim 135:226-231.

Gabriel S, Lau RW, Gabriel C. 1996. The dielectric properties of biological tissues: III. parametric models for the dielectric spectrum of tissues. Phys Med Biol 41:2271-2293.

Gandhi OP, Lam MS. 2003. An on-site dosimetry system for safety assessment of wireless base stations using spatial harmonic components. IEEE Trans Antennas Propag 51:840-847.

Habachi AE, Conil E, Hadjem A, Vazquez E, Wong MF, Gati A, Fleury G, Wiart J. 2010. Statistical analysis of whole-body absorption depending on anatomical human characteristics at a frequency of 2.1 GHz. Phys Med Biol 55:18751887 .

International Electrotechnical Commission (IEC). 2005. Human exposure to radio frequency fields from hand-held and body-mounted wireless communication devices - human models, instrumentation, and procedures to determine the specific absorption rate (SAR) for handheld devices used in the close proximity to the ear (frequency range of $300 \mathrm{MHz}$ To 3 $\mathrm{GHz}$ ). International Standard 62209. Geneva, Switzerland.

IEEE SCC39. 2005. IEEE Standard for Safety Levels with Respect to Human Exposure to Radio Frequency Electromagnetic Fields, 3 $\mathrm{kHz}$ to $300 \mathrm{GHz}$. IEEE C95.1-2005. New York, USA.
IEEE SCC28. 2002. IEEE Recommended Practice for Measurements and Computations of Radio Frequency Electromagnetic Fields With Respect to Human Exposure to Such Fields, 100 kHz-300 GHz. IEEE C95.3-2002. New York, USA.

International Commission on Non-Ionizing Radiation Protection (ICNIRP). 1998. Guidelines for limiting exposure to time-varying electric, magnetic, and electromagnetic fields. Health Phys 74:494-522.

Iskra S, McKenzie R, Cosic I. 2011. Monte-Carlo simulations of the electric field close to the body in realistic environments for application in personal radiofrequency dosimetry. Radiat Prot Dosimetry 147:517-527.

Joseph W, Verloock L and Martens L. 2006. Reconstruction of the Polarization Ellipse of the EM Field of Telecommunication and Broadcast Antennas by a Fast and Low-Cost Measurement Method. IEEE Trans Electromagn Compat 48:385-396.

Kientega T, Conil E, Hadjem A, Richalot E, Gati A, Wong MF, Picon O, Wiart J. 2011. A surrogate model to assess the whole body SAR induced by multiple plane waves at $2.4 \mathrm{GHz}$. Annals of Telecommunications 66:419-428.

Kühn S, Jennings W, Christ A, Kuster N. 2009. Assessment of induced radio-frequency electromagnetic fields in various anatomical human body models. Phys Med Biol 54:875-890.

Lazzi G, Gandhi O. 2000. A Mixed FDTD-Integral Equation Approach for On-Site Safety Assessment in Complex. IEEE Trans. Antennas Propag. 48:1830-1836.

Neubauer G, Preiner P, Cecil S, Mitrevski N, Gonter J, Garn H. 2009. The relation between the specific absorption rate and electromagnetic field intensity for heterogeneous exposure conditions at mobile communications frequencies. Bioelectromagnetics 30:651-662.

Olivier C. 2007. Characterisation of the electromagnetic radiation close to broadcast and wireless communications antennas. $\mathrm{PhD}$ thesis, Ghent University, Ghent, Belgium. 
Saunders SR, Aragon-Zavala A. 2007. Antennas and Propagation for Wireless Communication Systems (2nd ed.). Chichester: John Wiley \& Sons.

Taylor BN, Kuyatt CE. 1994. Guidelines for Evaluating and Expressing the Uncertainty of NIST Measurement Results. NIST Technical Note 1297. Gaithersburg, MD, USA.

Uusitupa T, Laakso I, Ilvonen S, Nikoskinen K. 2010. SAR variation study from 300 to 5000 $\mathrm{MHz}$ for 15 voxel models including different postures. Phys Med Biol 55:1157-1176.

Vermeeren G, Joseph W, Olivier C, Martens L. 2008a. Statistical multipath exposure of a human in a realistic electromagnetic environment. Health Phys 94:345-354.

Vermeeren G, Joseph W, Martens L. 2008b. Whole-body SAR in spheroidal adult and child phantoms in realistic exposure environment. Electron Lett 44:790-791.

Vermeeren G, Gosselin MC, Kühn S, Kellerman V, Hadjem A, Gati A, Joseph W, Wiart J, Meyer F, Kuster N, Martens L. 2010. The influence of the reflective environment on the absorption of a human male exposed to representative base station antennas from $300 \mathrm{MHz}$ to 5 GHz. Phys Med Biol 55:5541-5555. 